\title{
UTILIZACIÓN DE FACTORES DE CRECIMIENTO PLAQUETARIOS AUTÓLOGOS EN ARTRODESIS LUMBARES POSTEROLATERALES
}

\author{
UTILIZAÇÃO DE FATORES DE CRESCIMENTO PLAQUETÁRIOS AUTÓLOGOS \\ EM ARTRODESE LOMBAR PÓSTERO-LATERAL \\ USE OF AUTOLOGOUS PLATELET GROWTH FACTORS IN POSTEROLATERAL \\ LUMBAR SPINAL FUSIONS
}

Matías Andrés Rojas ${ }^{1}$, Natalia Roura², Pablo Marino²

\begin{abstract}
RESUMEN
Objetivo: Evaluar calidad e índice de pseudoartrosis en la formación del callo óseo en artrodesis instrumentadas lumbares posteriores con injerto óseo autólogo enriquecido con factores de crecimiento plaquetarios (FCP). Métodos: Estudio clínico analítico experimental simple ciego entre Junio de 2007 y Junio de 2009 comparando dos grupos, uno en el que se utilizó FCP en artrodesis lumbares posteriores y otro en el que no se aplicó el factor, evaluando las siguientes variables: índice de pseudoartrosis, formación del callo óseo y complicaciones postoperatorias. Resultados: Se evaluaron 26 pacientes, edad promedio 61,23 años, divididos en dos grupos de 13 pacientes. El grupo en el que se utilizó FCP presentó fusiones radiográficamente sólidas en el 61,54\%, sin registro de pseudoartrosis pero con incidencia de infección y sangrado postoperatorios de 15.39\%, mientras que en el grupo sin FCP el 69.23\% alcanzó fusiones sólidas con 7.69\% de pseudoartrosis sin otras complicaciones relacionadas con la intervención. Conclusión: Si bien el grupo con FCP no presentó pseudoartrosis, registró mayor incidencia de complicaciones postoperatorias y menor número de fusiones radiográficamente sólidas. Aunque la cantidad de pacientes no permite obtener resultados estadísticamente significativos el presente puede ser el inicio de un estudio mayor.
\end{abstract}

Palabras Claves: Factor de crecimiento derivado de plaquetas; Artrodesis; Pseudoartrosis.

\section{RESUMO}

Objetivo: Avaliar a qualidade e taxa de pseudoartrose em formação de calos em instrumentado fusão lombar com enxerto ósseo autólogo depois enriquecido com fatores de crescimento plaquetário (FCP). Métodos: Analytical experimental tipo cego única clínica entre junho de 2007 e junho de 2009 comparando dois grupos, um que foi usado no FCP após artrodese lombar e outro que não se aplicam, avaliar as seguintes variáveis : taxa de pseudoartrose de formação de calos e as complicações pós-operatórias. Resultados: Foram avaliados 26 pacientes, com idade média de 61,23 anos, divididos em dois grupos de 13 pacientes. O grupo que foi usado FCP apresentou fusões radiograficamente sólidos em 61,54\%, sem registro, mas com incidência não-união de infecção e sangramento pós-operatório 15,39\%, enquanto que no grupo sem o FCP\% 69,23 alcançado fusões sólidas com 7,69\% de pseudoartrose sem outras complicações relacionadas com a intervenção. Conclusão: Apesar de o grupo não se apresentar com FCP não sindicalizados, maior incidência de complicações pós-operatórias e menos fusões radiograficamente sólidos. Embora o número de pacientes que não é possível obter resultados estatisticamente significativos isso pode ser o início de um estudo maior.

Descritores: Fator de crescimento derivado de plaquetas; Artrodese; Pseudoartrose.

\begin{abstract}
Objective: To evaluate quality and rate of pseudoarthrosis in callus formation in instrumented posterolateral lumbar fusion with the use of autologous bone graft enriched with platelet growth factors (PGFs). Methods: A clinical analytical experimental single blind trial was carried out between June 2007 and June 2009 comparing two groups, one in which PGFs were used in lumbar spinal fusions while in the other the PGFs were not applied, to analyze the following variables: rate of pseudoarthrosis, callus formation and postoperative complications. Results: We evaluated 26 patients, mean age 61.23 years, divided in two groups of 13 patients each. The group in which PGFs were used presented radiographically solid fusions at $61.54 \%$ with no record of pseudoarthrosis, although with incidence of infection and postoperative bleeding of $15.39 \%$, while in the group without PGFs $69.23 \%$ achieved solid fusions presenting a rate of pseudoarthrosis of $7.69 \%$ with no other complications related to the intervention. Conclusion: Even though the group without PGFs did not present pseudoarthrosis, a higher incidence of postoperative complications and fewer radiographically solid fusions could be observed. Even though the number of patients is not sufficient to obtain statistically significant results, the present analysis may be the beginning of further study.
\end{abstract}

Keywords: Platelet-derived growth factor; Arthrodesis; Pseudarthrosis.

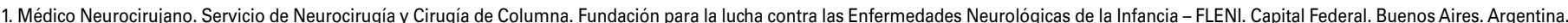

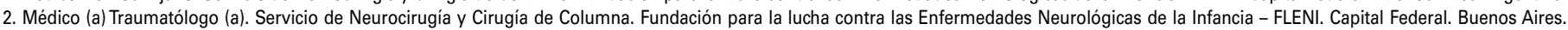
Argentina.

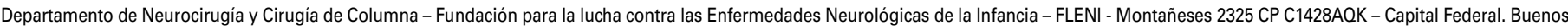
Aires. Argentina

Correspondência: Montañeses 2325 CP C1428AOK - Capital Federal - Buenos Aires, Argentina. Email: dr.marojas@ gmail.com 


\section{INTRODUCCIÓN}

La técnica de fusión lumbar ha sido durante décadas el tratamiento de elección para cuadros de dolor mecánico, trastornos degenerativos, deformidades progresivas, inestabilidad y trauma. En el caso de enfermedades degenerativas continúa siendo el procedimiento terapéutico más utilizado en el mundo. En los últimos años se han desarrollado numerosas técnicas con el objetivo de aumentar el índice de fusión y lograr mayor solidez de las mismas, incluyendo innovaciones en la exposición quirúrgica, técnica, instrumentación y, especialmente, aquellas relacionadas con el uso de injerto óseo y factores de crecimiento.

El injerto óseo autólogo de cresta ilíaca resulta el Gold Standard frente a los demás métodos de injerto ya que, además de poseer las propiedades requeridas en todo injerto viable de ser osteogénico, osteoconductivo y osteoinductivo, presenta la ventaja de su histocompatibilidad al provenir del propio paciente, reduciendo así riesgos de reacciones inmunológicas, transmisión de enfermedades y altos costos económicos.

A pesar de esto, la incidencia de pseudoartrosis afecta entre el 10 y $15 \%$ de las fusiones lumbares. Frente a estos casos y con el objetivo de prevenirlos, en la última década se ha promovido, entre otros procedimientos, el enriquecimiento del injerto con factores de crecimiento plaquetarios (FCP) que podrían potenciar la condición osteoinductora del injerto y mejorar su capacidad de adherencia y reparación ${ }^{1}$.

El objetivo de este trabajo es presentar nuestra experiencia con el uso de FCP en artrodesis lumbares instrumentadas posteriores en una serie de pacientes en los que se evaluó, principalmente, la calidad y velocidad de formación del callo óseo e índice de pseudoartrosis.

\section{MÉTODOS}

Se llevó a cabo un estudio analítico experimental de tipo ensayo clínico aleatorio que incluyó a los pacientes tratados en nuestra institución, FLENI (Fundación para la lucha contra las Enfermedades Neurológicas de la Infancia), entre Junio de 2007 y Junio de 2009 con artrodesis lumbares instrumentadas posteriores comparando la evolución entre el grupo resultante de pacientes en los que se indicó en forma controlada y aleatoria la utilización de FCP, a modo de enriquecimiento del injerto óseo, y el grupo restante de pacientes en el que los mencionados factores no fueron aplicados. Con el fin de evitar que el conocimiento de la técnica pudiera influir de alguna manera en la evolución de los pacientes se utilizaron técnicas de enmascaramiento tornando al ensayo clínico "simple ciego" de modo que los pacientes en los que se utilizaron los FCP ignoraban esta situación.

El estudio comprendió 26 pacientes de edad promedio 61,23 años (rango etario: 30-87 años), divididos en dos grupos de 13 pacientes. El grupo en el que se empleó FCP, integrado por 8 hombres $(61,54 \%)$ y 5 mujeres $(38,46 \%)$, presentaba una edad promedio de 61,62 años (rango: 30-87 años) mientras que el grupo sin FCP se integraba por 9 hombres $(69,23 \%)$ y 4 mujeres $(30,77 \%)$ y presentaba una edad promedio de 60,85 años (rango: 34-82 años). La proporción de pacientes mayores de 65 años resultó de un 54\% para ambos grupos, edad que se tomó como punto de corte y a la que se le atribuyó expresa valoración dado que la edad avanzada es un factor que puede afectar la correcta consolidación del callo óseo.

Entre los antecedentes médicos relevantes al estudio cabe destacar la presencia en ambos grupos de sobrepeso (BMI>27) en un $38,46 \%$ (5 pacientes) y diabetes y/o insulino-resistencia en un $7,69 \%$ (1 paciente). Así como el estado nutricional y ciertas enfermedades metabólicas resultan otros factores influyentes en la consolidación ósea, también lo son las enfermedades sistémicas de afección ósea como la osteoporosis y la artritis reumatoidea que fueron encontradas solamente en el grupo con FCP en un 7,69\% cada una (1 paciente). Con igual incidencia en este grupo, se halló el antecedente de Enfermedad de Parkinson que puede influir en la etapa de remodelación ósea, que constituye la fase final y tardía en la formación del callo.
Está comprobado que la ingesta de anti-inflamatorios no esteroideos (AINES) afecta la etapa de organización temprana de fusión ósea. En el grupo con FCP el 61,54\% (8 pacientes) consumían habitualmente AINES mientras que en el grupo sin FCP lo hacía el 46,15\% (6 pacientes) (Figura 1 y 2). Por su parte, el tabaquismo influye negativamente en la etapa proliferativa o intermedia de formación del callo. El 38,46\% (5 pacientes) del grupo con FCP eran tabaquistas mientras que en el grupo sin FCP sólo el 7,69\% (1 paciente) (Figura 3 y 4 ).

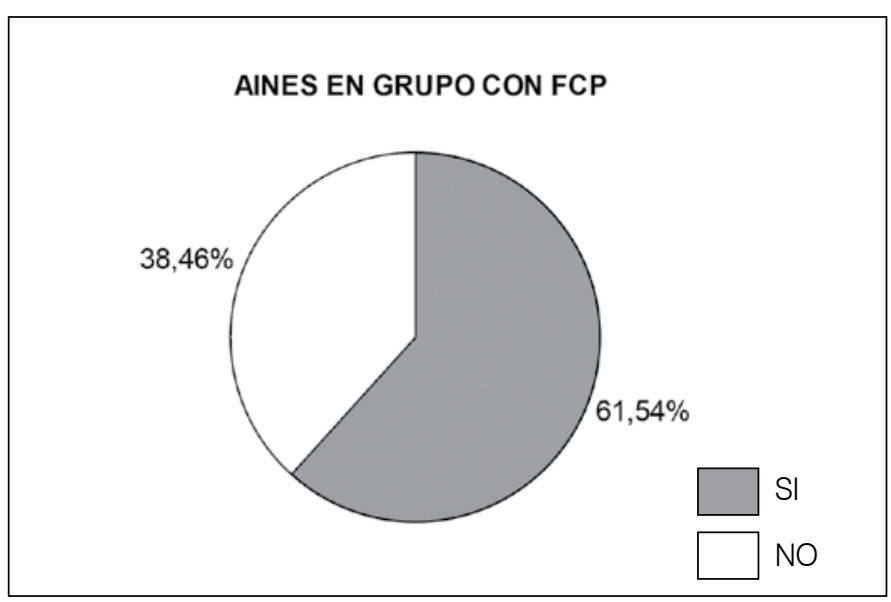

Figura 1. Consumo de AINES en GRUPO CON FCP.

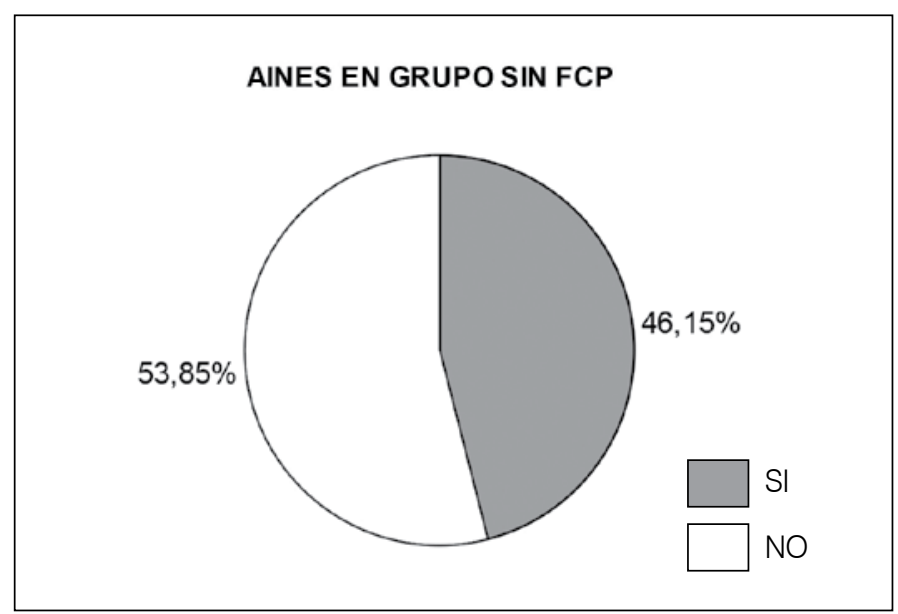

Figura 2. Consumo de AINES en GRUPO SIN FCP.

TABAQUISMO EN GRUPO CON FCP

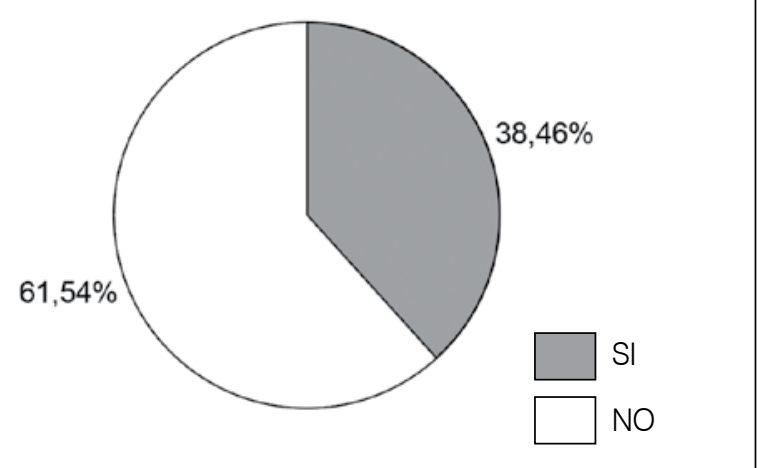

Figura 3. Tabaquismo en GRUPO CON FCP. 


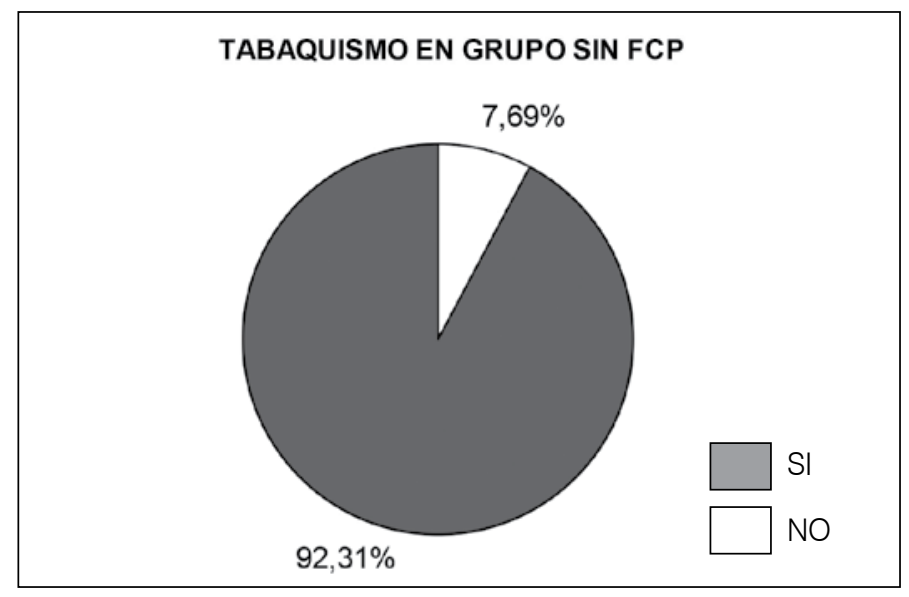

Figura 4. Tabaquismo en GRUPO SIN FCP.

Ambos grupos presentaban el antecedente de cirugías espinales previas en 6 pacientes (46,15\%), de los cuales, y también en ambas muestras, 3 pacientes (23,08\% del total de cada grupo) habían sido sometidos a artrodesis instrumentadas previas y el resto a cirugías menores, principalmente discectomías y colocación de dispositivos inter-espinosos de evolución fallida.

En el grupo con FCP los diagnósticos que dieron lugar a la indicación quirúrgica de artrodesis lumbar posterior fueron los siguientes: canal estrecho degenerativo en 3 pacientes $(23,08 \%)$, espondilolistesis en 5 pacientes (38,46\%), escoliosis degenerativa en 2 pacientes (15,38\%), escoliosis asociada a canal estrecho en 2 pacientes $(15,38 \%)$ y espondilolísis con necesidad de extracción del dispositivo inter-espinoso previo en 1 paciente (7,69\%) (Figura 5).

Por su parte, en el grupo sin FCP los diagnósticos que llevaron a la cirugía fueron los siguientes: canal estrecho degenerativo en 2 pacientes (15,38\%), espondilolistesis en 4 pacientes $(30,77 \%)$, pseudoartrosis de artrodesis instrumentada previa con necesidad de revisión y recambio de la misma en 2 pacientes (15,38\%), escoliosis asociada a canal estrecho en 1 paciente $(7,69 \%)$, síndrome de segmento supra-adyacente a artrodesis instrumentada previa con necesidad de prolongación de la misma en 1 paciente $(7,69 \%)$, fractura vertebral en 2 pacientes (15,38\%) y escoliosis asociada a canal estrecho y espondilolistesis en 1 paciente (7,69\%) (Figura 6).

En todos los pacientes estudiados se realizó artrodesis instrumentada posterolateral inter-transversa con injerto autólogo de cresta ilíaca. En el grupo con FCP se realizó fusión mayor a dos niveles vertebrales en el 53,85\% (7 pacientes), fusión de dos niveles en el $30,77 \%$ (4 pacientes) y de un solo nivel en el $15,38 \%$ ( 2 pacientes) (Figura 7). A su vez, los niveles fusionados con mayor frecuencia fueron L4 a S1 en el 30,77\% (4 pacientes), L2 a L5 y T11 a S1 en el 15,38\% (2 pacientes) cada uno y T11 a L5, L1 a L5, L1 a S1, L4 a L5 y L5 a S1 en un 7,69\% (1 paciente) cada uno (Figura 8).

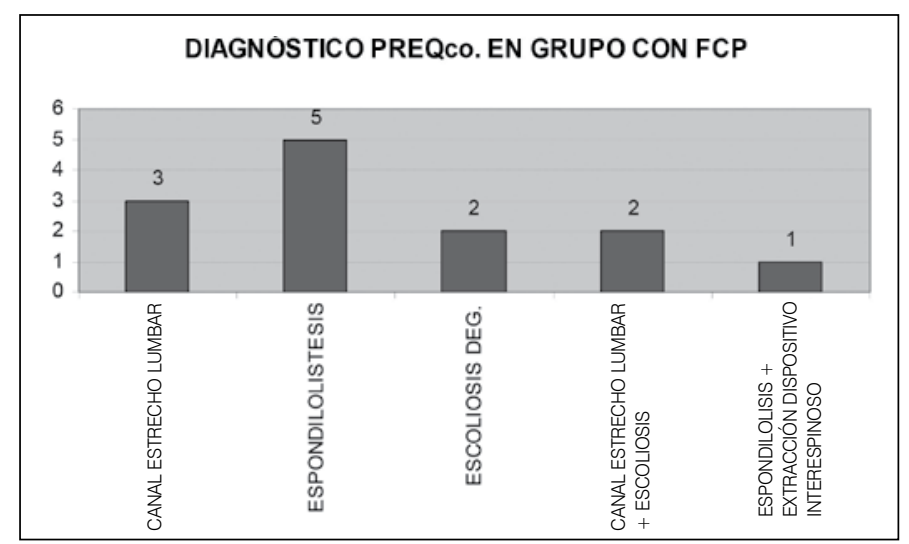

Figura 5. Diagnósticos pre quirúrgicos en GRUPO CON FCP.
En el grupo sin FCP se realizó fusión mayor a dos niveles vertebrales en el 46,15\% (6 pacientes), fusión de dos niveles en el $38,46 \%$ (5 pacientes) y de un solo nivel en el $15,38 \%$ (2 pacientes) (Figura 9). Los niveles L4 a S1 fueron fusionados en el 30,77\% de la muestra (4 pacientes), L2 a S1 y L4 a L5 en el 15,38\% (2 pacientes) cada uno y T9 a L2, T11 a S1, T12 a L4, L3 a L5 y L3 a S1 en el 7,69\% (1 paciente) cada uno (Figura 10).

Todos los pacientes del estudio fueron evaluados clínica e imagenológicamente en forma periódica a los tres, seis y doce meses postoperatorios sin perder ningún paciente en el seguimiento. El estudio se centró en las siguientes variables: índice de pseudoartrosis, formación del callo óseo (evaluando su tamaño y densidad) y beneficios secundarios de los FCP frente a complicaciones postoperatorias (disminución de sangrado, dolor, infección y colección de seromas).

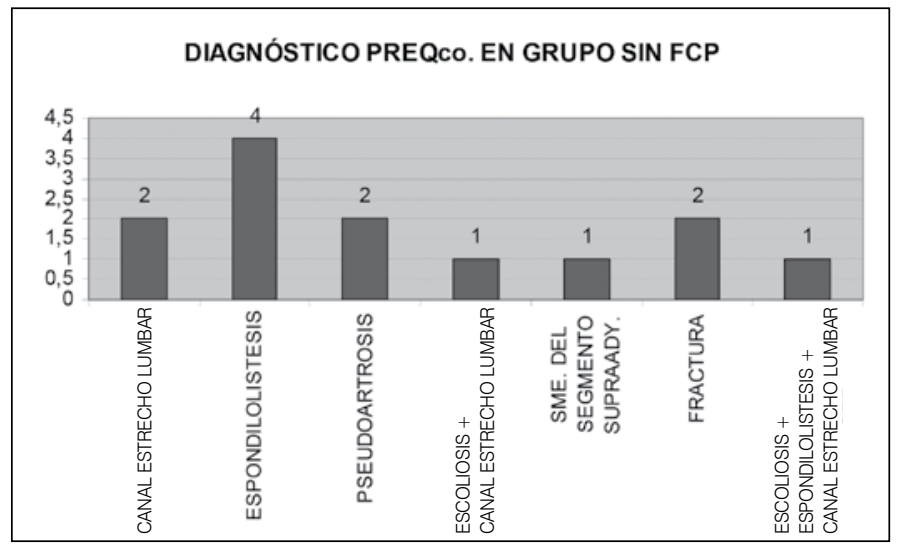

Figura 6. Diagnósticos pre quirúrgicos en GRUPO SIN FCP.

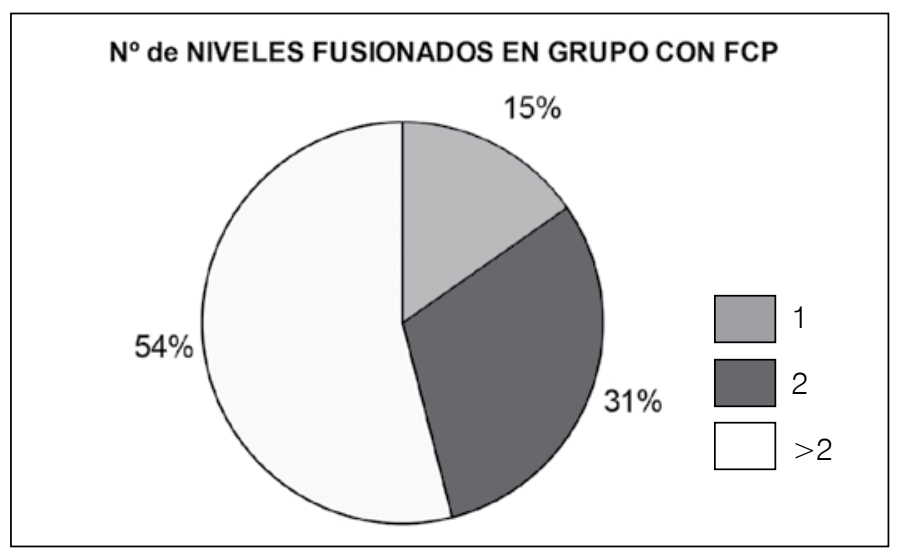

Figura 7. Cantidad de niveles fusionados en GRUPO CON FCP.

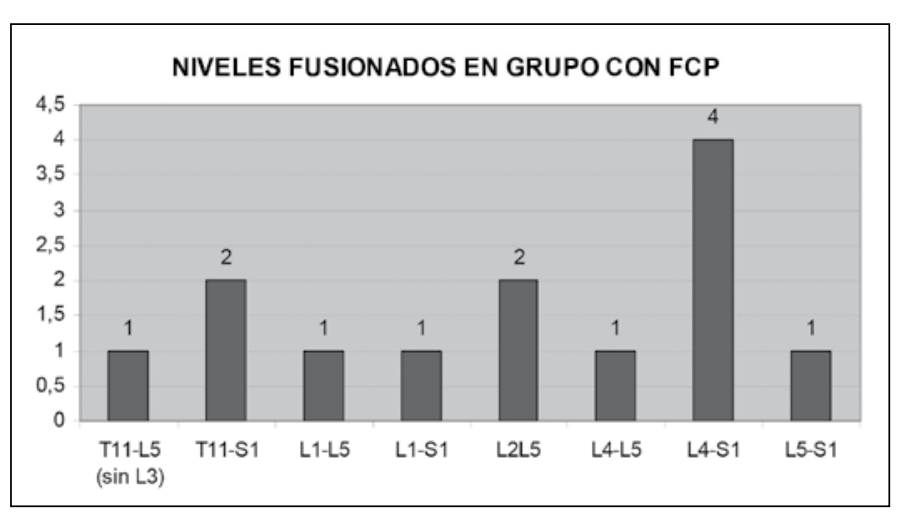

Figura 8. Niveles fusionados en GRUPO CON FCP. 


\section{$N^{\circ}$ de NIVELES FUSIONADOS EN GRUPO SIN FCP}
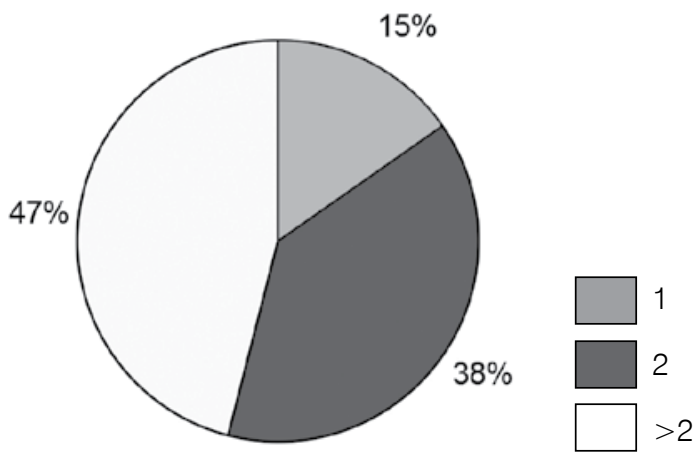

Figura 9. Cantidad de niveles fusionados en GRUPO SIN FCP.

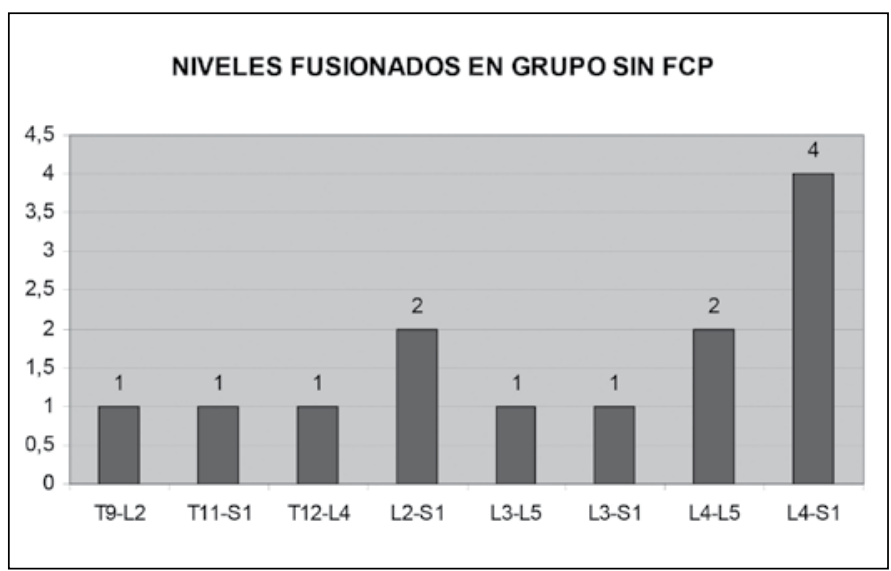

Figura 10. Niveles fusionados en GRUPO SIN FCP.

\section{TÉCNICA QUIRÚRGICA}

Consiste en un abordaje sobre línea media de la columna en pacientes en decúbito ventral con abdomen libre exponiendo de forma subperióstica las láminas, facetas articulares, apófisis transversas y, en algunos casos, el alerón sacro, para proceder a una instrumentación con tornillos transpediculares y barras bilaterales, de acuerdo a la cantidad de niveles fusionados, y a una artrodesis ósea con injerto de cresta ilíaca autólogo en el espacio de las apófisis transversas tras haber sido decorticadas. En los pacientes seleccionados, se enriqueció el injerto con la aplicación local de FCP en forma local.

\section{Técnica de preparación del injerto óseo}

El injerto de hueso esponjoso se obtiene de las crestas ilíacas posteriores del paciente al inicio del acto quirúrgico y es procesado para eliminar las corticales y los tejidos blandos, para luego triturarlo hasta lograr un microgranulado de consistencia pastosa.

\section{Técnica de preparación del concentrado de FCP}

Se obtiene una unidad de sangre (450-500 cc) del paciente previamente al inicio de la cirugía. Esta muestra es sometida a centrifugación obteniendo tres interfaces: un sobrenadante plasmático pobre en plaquetas, un precipitado de glóbulos rojos y una fase fina y blanca en el medio de las anteriores ("buffy coat") rica en plaquetas. Mientras las dos primeras son retransfundidas al paciente, la última fase es sometida a ultra centrifugación para obtener finalmente un concentrado de FCP que es agregado al injerto de hueso esponjoso obtenido al inicio de la cirugía junto con 3-6 cc de trombina para la formación de un gel aplicable en los espacios de apófisis transversas vertebrales decorticadas.

\section{Seguimiento Clínico}

Los pacientes fueron seguidos en el postoperatorio por intervalos programados de 3, 6 y 12 meses en nuestra institución. Todos los pacientes fueron interrogados con respecto a su evolución y se constató el correcto uso en el postoperatorio inmediato, mediato y alejado (hasta tres meses en la mayoría de los casos) de la ortosis lumbosacra indicada en la totalidad de los casos para proteger la fusión de movimientos excesivos y facilitar la formación del callo óseo. La aparición de dolor lumbar y/o radicular en los controles postoperatorios alejados ambulatorios promovió la realización de estudios imagenológicos que pudieran confirmar o descartar la presencia de pseudoartrosis.

\section{Seguimiento y manejo imagenológico}

En cada instancia de control clínico se realizaron en todos los pacientes radiografías y tomografías axiales computadas con reconstrucción 2D sagital y coronal, evaluadas en conjunto con el servicio de "Diagnóstico por Imágenes" considerando los siguientes parámetros de estudio:

Radiografías: Se tomaron incidencias lumbares antero-posteriores con el fin de evaluar el tamaño de callo óseo formado en las artrodesis, estableciendo la siguiente clasificación imagenológica de fusiones óseas:

Tipo I: Fusiones "sólidas" con callo óseo que excede las apófisis transversas (Figura11).

Tipo II: Fusiones "suficientes" con callo óseo que alcanza a las apófisis transversas en sus límites laterales externos sin excederlas (Figura 12).

Tipo III: Fusiones "insuficientes" con callo óseo no visible o escaso entre las apófisis transversas (Figura 13).

Tomografía: A manera de evaluar la densidad del callo óseo formado se realizaron mediciones densitométricas tomográficas en unidades Hounsfield $(\mathrm{UH})$ comparando el valor de las mismas a nivel del callo óseo de la artrodesis con otras tomadas en áreas articulares vertebrales del mismo paciente no comprometidas por el acto quirúrgico, tomando éste último valor como parámetro de la calidad basal de densidad ósea del individuo estudiado. Así, una densidad del callo óseo por debajo de ese valor basal se consideró como "callo óseo inadecuado", mientras que si resultaba por encima del mismo se lo interpretó como "callo óseo sólido" (Figura 14).

Asimismo y a partir de ambos estudios, se definió la presencia de pseudoartrosis ante la evidencia de una solución de continuidad en la trama reticular del callo óseo y/o cavitación del material de osteosíntesis con o sin signos de inestabilidad (Figura 15).

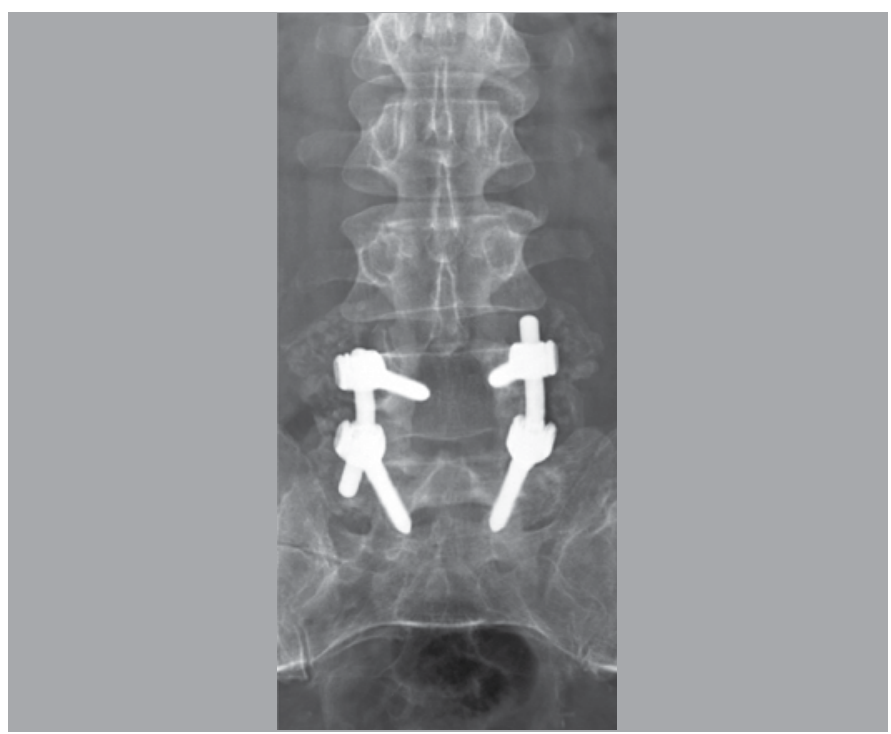

Figura 11. Fusión ósea tipo I. 


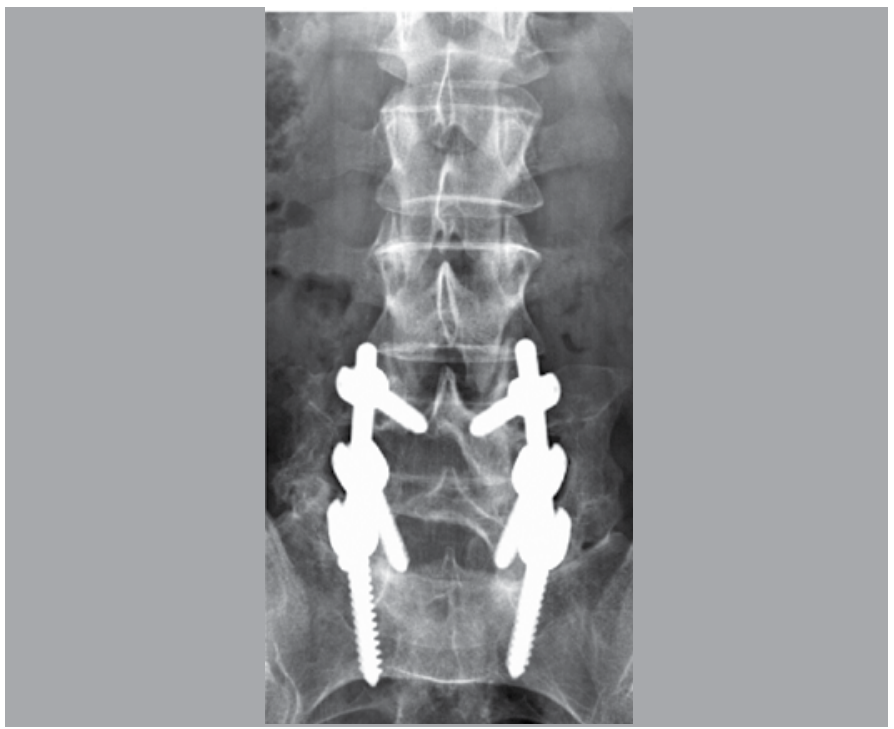

Figura 12. Fusión ósea tipo II.

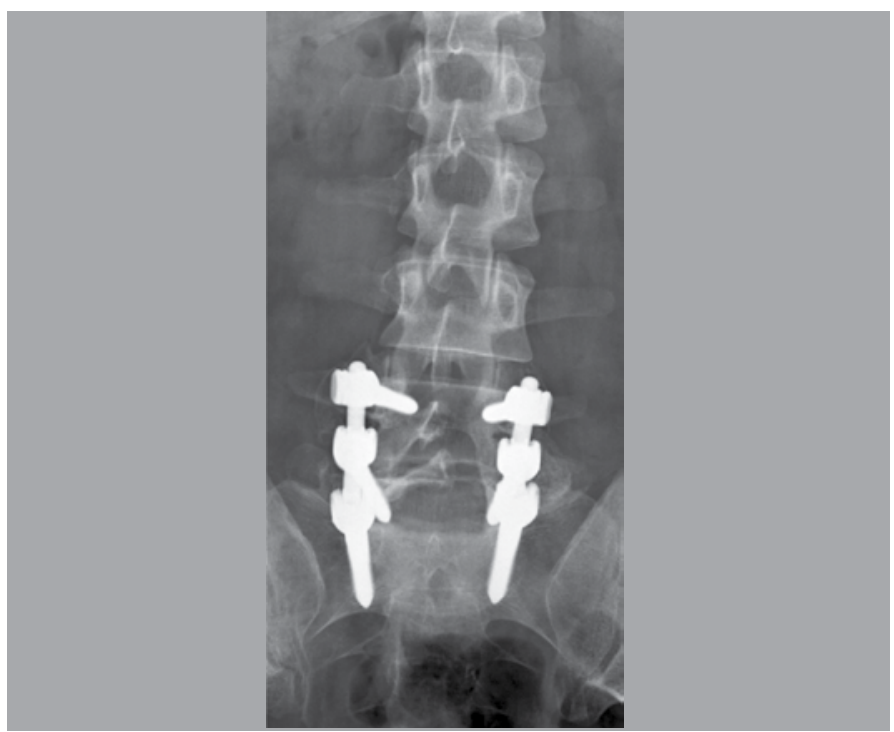

Figura 13. Fusión ósea tipo III.

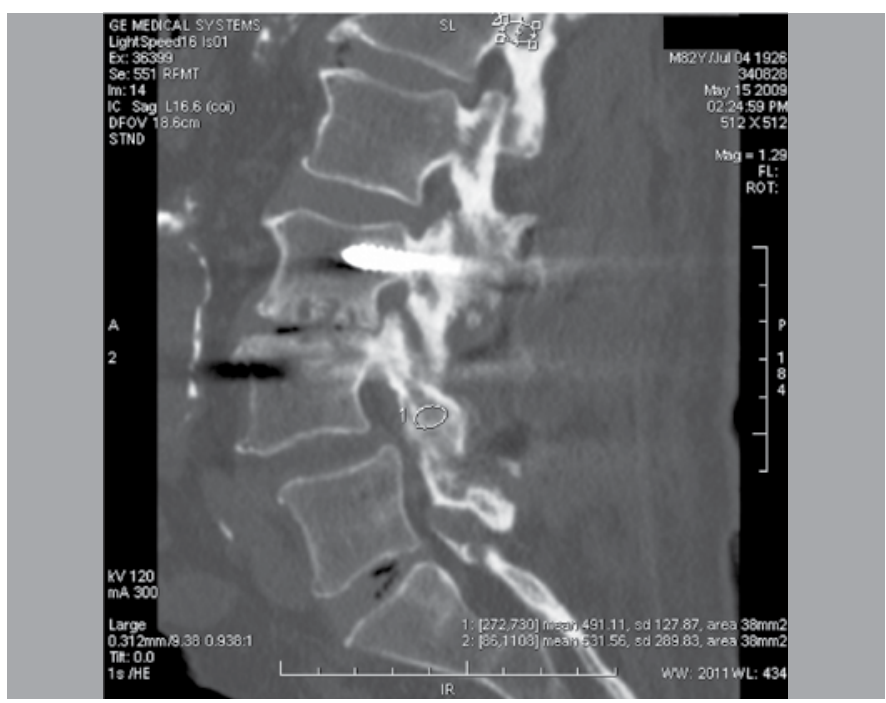

Figura 14. TAC con medición de densidades óseas a nivel de áreas marcadas circulares como regiones de interés (ROI 1 y ROI 2) en reconstrucción 2D sagital.

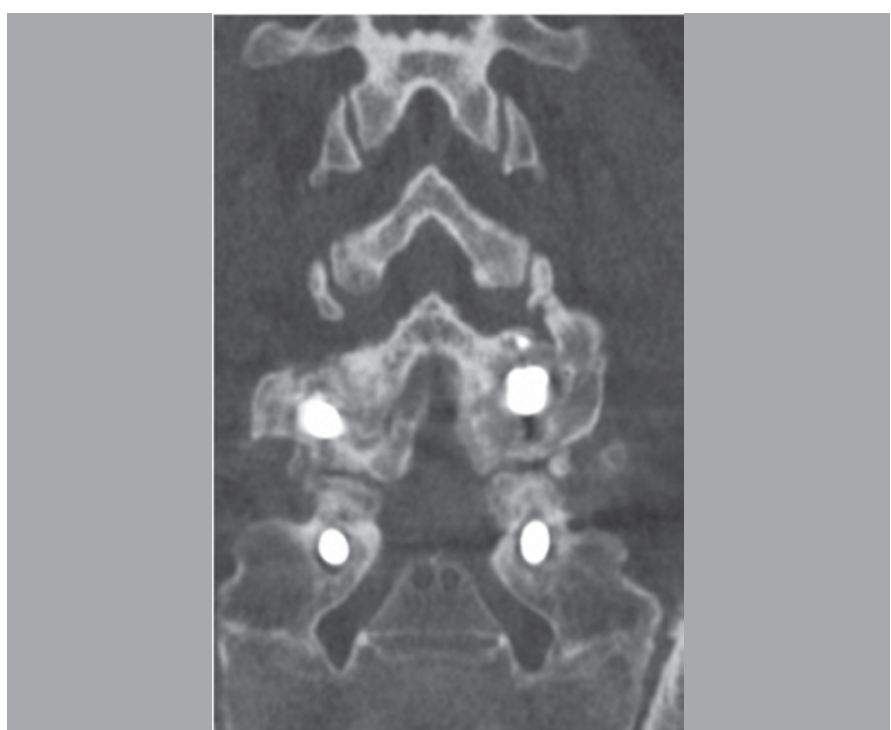

Figura 15. TAC con reconstrucción $2 \mathrm{D}$ coronal. Pseudoartrosis: Soluciones de continuidad en callos óseos bilaterales.

\section{RESULTADOS}

En nuestras series, y en contraposición a lo esperado, el grupo con FCP logró sólo en un 61,54\% (8 de los 13 pacientes) alcanzar en los controles radiográficos fusiones óseas sólidas tipo I, mientras que el grupo sin FCP alcanzo este grado de fusión en un $69,23 \%$ (9 de los 13 pacientes). Las fusiones óseas tipo II fueron evidenciadas en un 23,08\% (3 pacientes) del grupo con FCP y en un $7,69 \%$ (1 paciente) del grupo sin FCP y las fusiones tipo III en un $15,38 \%$ (2 pacientes) y en un $23,08 \%$ (3 pacientes) en respectivas muestras (Figura 16)

En cuanto a las mediciones de densidades óseas tomográficas, el grupo con FCP logró fusiones óseas con densidades a niveles de los callos óseos en un promedio de 693,39 UH, que resultaron superiores a las densidades basales óseas promedio a otros niveles espinales de los mismos pacientes (418,03 UH), así como también superaron las densidades óseas logradas por las fusiones lumbares de los pacientes del grupo sin FCP que promediaban las 460,57 UH sobre densidades basales de 407,76 UH (Figura 17).

También resultó opuesta a la esperada la incidencia de complicaciones postoperatorias, que resultaron predominantes en el grupo con FCP. En éste, se presentaron sangrados importantes a nivel del sitio quirúrgico que requirieron desde transfusión de unidades de glóbulos rojos por anemia marcada hasta intubación orotraqueal en unidad de cuidados intensivos por shock hipovolémico en 2 pacientes $(15,38 \%)$ e infección postoperatoria de sitio quirúrgico con aislamiento de Staphylococcus Aureus meticilino sensible y Staphylococcus Epidermidis con requerimiento de tratamiento antibiótico endovenoso también en 2 pacientes (15,38\%). En ambos grupos, un sólo paciente $(7,69 \%)$ presentó en el postoperatorio inmediato coagulopatía por consumo con requerimiento de poli-transfusiones de plaquetas y glóbulos rojos, resultando la única complicación postoperatoria en el grupo sin FCP (Figura 18).

La necesidad de permanencia de drenajes quirúrgicos fue en promedio de 2,15 días en el grupo con FCP y 2 días en el grupo sin FCP, con un promedio de débito por los mismos de 484,61 ml y $685,13 \mathrm{ml}$ respectivamente. No se constataron seromas ni otras colecciones postquirúrgicas relevantes en ninguno de los grupos.

Los pacientes del grupo con FCP requirieron morfina, o derivados de la misma, para paliar el dolor en el postoperatorio inmediato y mediato por un promedio de 1,54 días, mientras que en el grupo sin FCP la necesidad de analgesia opiácea promedió los 1,85 días.

A pesar de la mayor incidencia de complicaciones postoperatorias inmediatas y mediatas en el grupo con FCP, este requirió un promedio de 2,08 días de internación en UTI y 6,23 días en sala 


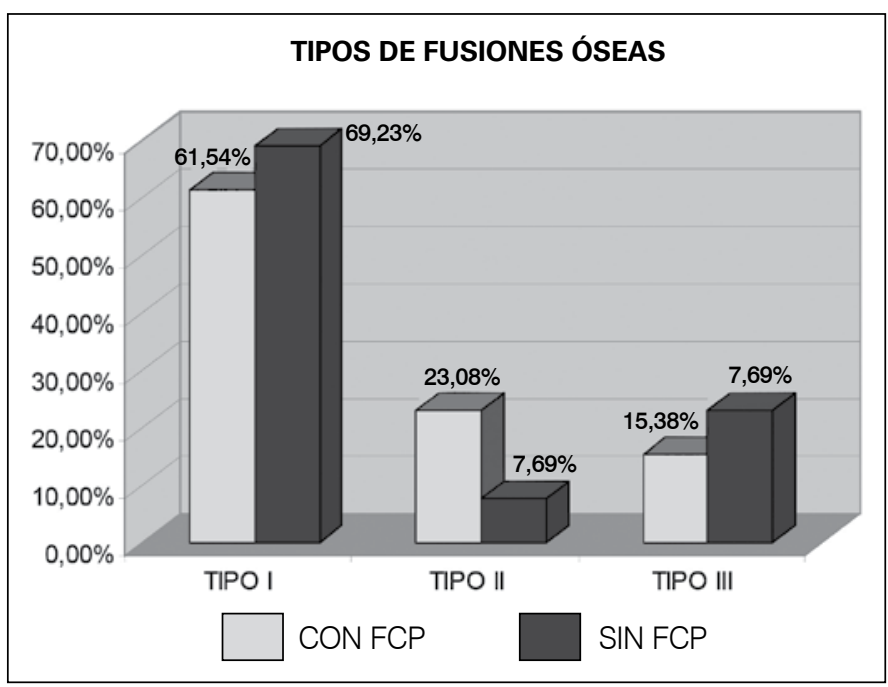

Figura 16. Incidencia de los diversos tipos de fusiones óseas.

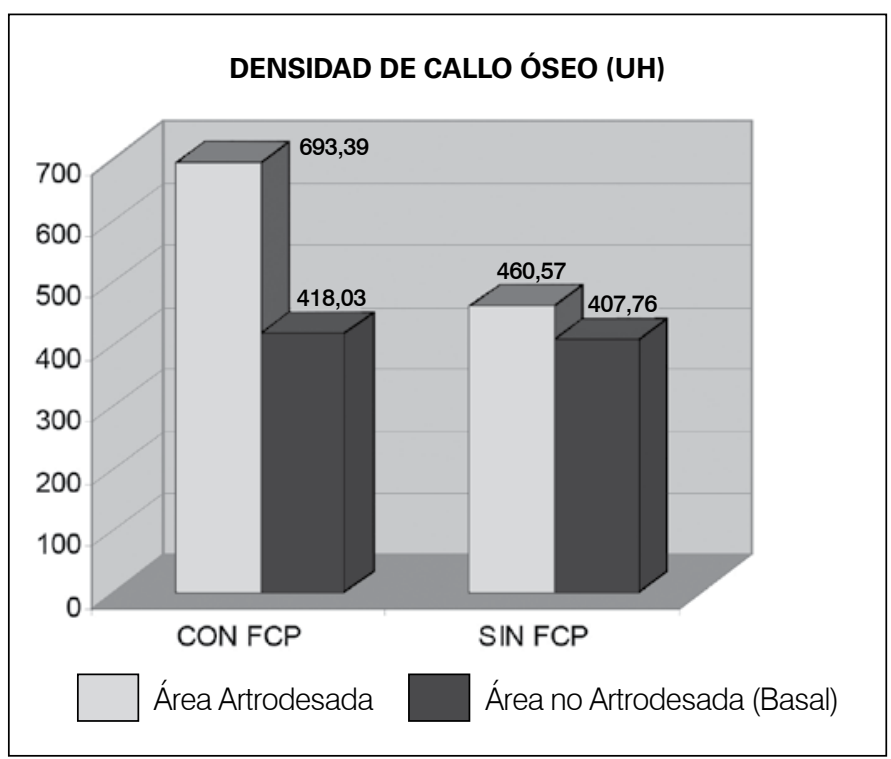

Figura 17. Promedios de densidades topográficas de callos óseos a nivel de las fusiones.

general, resultando internaciones menores a las promediadas para el grupo sin FCP (7 días en UTI y 2,23 días en sala general).

Finalmente en los controles postoperatorios alejados ambulatorios, se constató la presencia de pseudoartrosis tanto clínica como imagenológicamente (ausencia de consolidación ósea y persistencia de movilidad superando los 3-4 meses postoperatorios) en un sólo paciente perteneciente al grupo sin FCP (7,69\% del mismo) (Figura 19).

\section{DISCUSIÓN}

A pesar de las importantes modificaciones y mejorías en las técnicas quirúrgicas, tecnología de implantes y el uso de injertos óseos autólogos que han tenido lugar en los últimos años, un 5\% al 10\% de las artrodesis lumbares no logran consolidar y evolucionan a la pseudoartrosis. Las causas de este fenómeno son multifactoriales. El mejor entendimiento de los factores biológicos que intervienen en la osteogénesis y formación del callo óseo, que también se ha incrementado en los últimos tiempos, ha incentivado a investigadores a buscar formas de manipulación del ambiente biológico en el que la formación de nuevo hueso toma lugar con el fin de potenciar la fusión ósea.

La formación del callo óseo comprende tres etapas. La "eta-

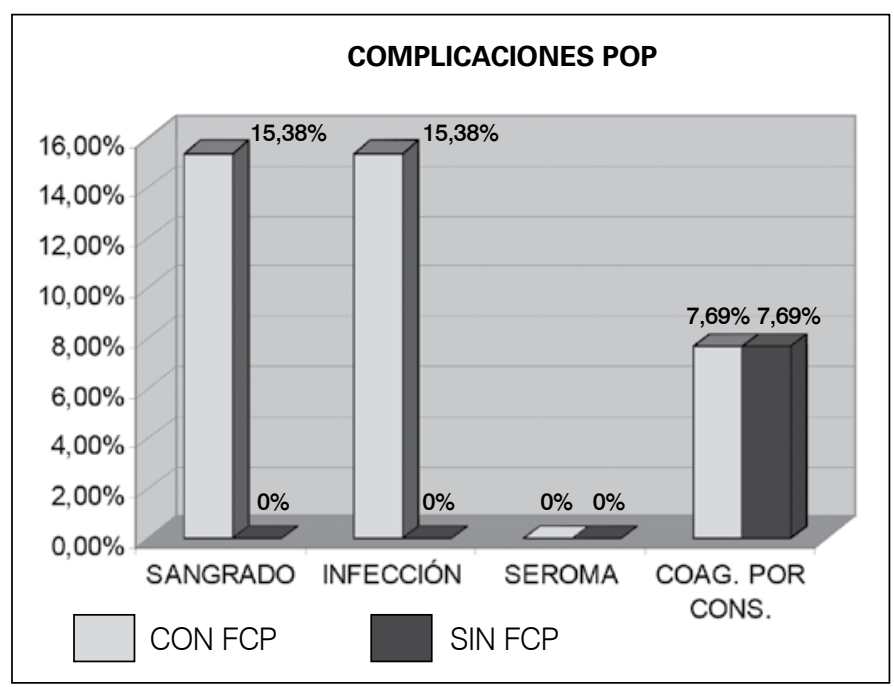

Figura 18. Complicaciones postoperatorias

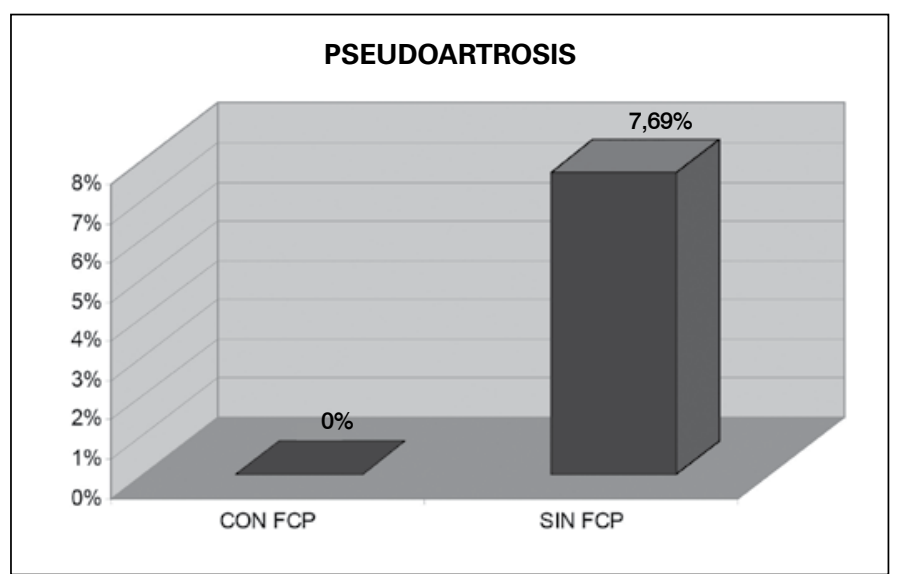

Figura 19. Incidencia de pseudoartrosis.

pa inflamatoria temprana" (o "de consolidación del hematoma") ocurre en las primeras 24 a 48 hs y se centra en la formación de tejido de granulación frente a la injuria del tejido óseo. Esta fase está mediada por la acción de prostaglandinas, razón por la cual la ingesta de AINES puede alterarla considerando sus conocidos efectos en la inhibición de varias vías de formación de las mismas. La "etapa de reparación ósea" (o "proliferativa") continúa a la anterior en las posteriores 4 semanas, durante las cuales los fibroblastos y células osteogénicas, principalmente, se encargan de formar el estroma que servirá de soporte para la revascularización de la zona afectada. Esta fase se ve afectada en pacientes tabaquistas como consecuencia de la injuria endotelial microvascular asociada a los componentes del tabaco. Finalmente, la "etapa de remodelación ósea" se desarrolla en los próximos 3 a 6 meses durante la cual se alcanza la estructura ósea final, producto del equilibrio entre la actividad osteoblástica y osteoclástica local y facilitada por el stress mecánico al que la zona está expuesta ${ }^{2,3}$. Considerando los procesos involucrados, resultan críticas las primeras dos semanas en la formación de un callo óseo adecuado.

Aparte de los factores biológicos naturales del sitio involucrado, existen factores externos locales, como las radiaciones e infecciones, y sistémicos asociados a la condición y hábitos del paciente que pueden afectar la consolidación del callo óseo. Dentro de los últimos, deben considerarse la edad y el estado nutricional del paciente, hábito de tabaquista o consumo de medicación (AINES, corticoides, agentes citotóxicos entre otros), alteraciones endocrinológicas (especialmente del metabolismo del calcio: vitamina D, 
calcitriol, calcitonina, hormona paratiroidea) y enfermedades metabólicas-óseas (diabetes, osteoporosis, artritis reumatoidea, etc.) ${ }^{4}$.

Entre los factores que estimularían y potenciarían la fusión y formación ósea se han identificado y cobrado notoriedad los factores de crecimiento secretados localmente en el lugar de injuria ósea, y entre ellos figuran aquellos producidos y liberados por las plaquetas. En los gránulos alfa plaquetarios se almacenan para su posterior liberación durante la "etapa inflamatoria temprana" de formación del callo óseo los siguientes factores ${ }^{3,5,6}$ :

- PDGF-a $\beta$, PDGF-aa y PDGF- $\beta \beta$ ("platelet derived growth factor" o "factor de crecimiento derivado de plaquetas"): polipéptido termoestable con peso molecular de 30.000 Daltons y constituido por dos cadenas de aminoácidos denominadas $A$ y $B$ que se unen a dos receptores tirosincinasa para generar una respuesta mitogénica en las células blanco. Además, poseen acción quimiotáctica sobre precursores osteoblásticos y angiogénica a través de la estimulación de macrófagos y células endoteliales en la formación de nuevos capilares.

- TGF- $\beta 1$, TGF- $\beta 2$, TGF- $\beta 3$ ("transforming growth factor beta" o "factor de crecimiento de transformación Beta"): proteínas de 25.000 Daltons que estimulan la formación ósea aumentando la proliferación de células progenitoras o "stem" al tiempo que inhiben la resorción ósea al inhibir los osteoclastos.

- VEGF - EGF - FGF ("factor de crecimiento del endotelio vascular", "factor de crecimiento epitelial", "factor de crecimiento fibroblástico"): polipéptidos encargados de controlar la proliferación y diferenciación fibroblástica así como de estimular la angiogénesis mediante la promoción de mitosis y migración de células endoteliales.

- IGF ("insulin-like growth factor" o "factor de crecimiento semejante a la insulina"): proteínas con estructura similar a la insulina en un 50\% y capacidad de estimular la síntesis de matriz ósea y la diferenciación de osteoblastos.

Las propiedades quimiotácticas sobre células involucradas en la inmunidad con acción anti-inflamatoria, angiogénicas y de estimulación epitelial y endotelial cicatrizante así como de la síntesis ósea, junto a la relativa facilidad de su obtención y su viabilidad económica han hecho que los FCP se impongan como una opción atractiva en el enriquecimiento del foco de consolidación de fusión ósea, ya sea solos o con el agregado de injerto. Sustentado en diversos trabajos científicos, el concentrado de factores plaquetarios aportaría los siguientes beneficios ${ }^{1,5,7,8}$

- Mejorarían y acelerarían la curación y cicatrización de heridas.

- Reducirían secreciones y sangrados postoperatorios.

- Reducirían inflamación local y dolor asociado y, por consiguiente,

la necesidad de opiáceos y demás analgésicos.

- Reducirían riesgo de infecciones postoperatorias.

- Como consecuencia de lo expuesto, reducirían la duración de internaciones hospitalarias.

- Mejorarían y acelerarían el proceso de consolidación del callo óseo.

En nuestras series de pacientes, y contrariamente a lo esperado, el grupo sin FCP alcanzó fusiones con callo óseo hipertrófico radiográficamente en mayor número que el grupo con FCP, resultando en un $69,23 \%$ de la muestra del primer grupo contra $61,54 \%$ del segundo $(p=1)$. Aún así, debería considerarse que en la mayor consolidación del callo óseo en el grupo sin FCP con respecto al grupo con FCP podría haber influido su bajo índice de población tabaquista, $38,46 \%$ de la primer muestra contra $7,69 \%$ de la segunda $(p=0,16)$, y de menor consumo de AINES, 46,15\% de la primer muestra contra

\section{REFERÊNCIAS}

1. Hee HT, Majd ME, Holt RT, Myers L. Do autologous growth factors enhance transforaminal lumbar interbody fusion? Eur Spine J. 2003:12(4):400-7.

2. Kalfas IH. Principles of bone healing. Neurosurg Focus. 2001;10(4):E1.

3. Pilitsis JG, Lucas DR, Rengachary SS. Bone healing and spinal fusion. Neurosurg Focus. 2002;13(6):e1.

4. Brinker MR, O'Connor DP, Monla YT, Earthman TP. Metabolic and endocrine abnormalities in patients with nonunions. J Orthop Trauma. 2007;21(8):557-70.

5. Casteulani A. C. Enriquecimiento de injerto autólogo con concentrado de factores de
$61,54 \%$ de la segunda $(p=0,43)$. Por otro lado, del total de pacientes del estudio que alcanzaron fusiones con callo óseo hipertrófico tipo I (17 de los 26 pacientes) sólo 11,76\% (2 pacientes) eran tabaquistas y el $23,07 \%$ (6 de los 26 pacientes) consumían AINES y eran mayores de 65 años. Asimismo, sólo un 20\% (1 de 5 pacientes) de la totalidad de pacientes que alcanzaron fusiones óseas insuficientes tipo III tenían como antecedentes osteoporosis, tabaquismo, consumo de AINES y edad superior a 65 años.

Si bien radiográficamente el grupo con FCP alcanzó fusiones ósea tipo I en menor número que el grupo sin FCP, tomográficamente el primer grupo logró mayor densidad ósea en las mismas que promediaban las 693,39 UH contra las 460,57 UH del segundo grupo.

Otro hallazgo del estudio, que también resultó contrario a lo previsto, fue la incidencia de complicaciones postoperatorias como sangrados e infecciones relacionadas con la cirugía en el grupo con FCP que abarcó para cada caso al 15,38\% (2 de 13 pacientes) de la muestra mientras que no hubo registros de las mismas en el grupo sin FCP $(p=0,46)$. De los pacientes que presentaron sangrados postoperatorios $50 \%$ eran tabaquistas y de aquellos con infección postoperatoria el 100\% consumían AINES. Asimismo, la consideración de la totalidad de complicaciones postoperatorias en ambos grupos afectaría a un 38,46\% del grupo con FCP (5 de 13 pacientes) lo que supera al 7,69\% (1 de 13 pacientes) de la población afectada en el grupo sin FCP.

En cuanto a las secreciones postoperatorias, el grupo con FCP promedió débitos por drenaje menores al grupo sin FCP en una diferencia de $200 \mathrm{ml}$ en las primeras 48 hs posteriores a la cirugía.

En lo que se refiere al manejo del dolor, no hubo prácticamente diferencia en la cantidad de días de requerimiento de opiáceos por parte de los dos grupos, como tampoco la hubo en la duración total de las internaciones, si bien el grupo sin FCP promedió una cantidad de días de internación en unidad de cuidados intensivos mayor a 3 veces de la que requirió el grupo con FCP, a pesar de la mayor tasa de complicaciones en éste último.

Se registró un solo caso en todo el estudio de pseudoartrosis en la artrodesis lumbar realizada correspondiente a 1 paciente que tenía como único antecedente el consumo de AINES perteneciente al grupo sin FCP, mientras que en el grupo con FCP no hubo evidencia clínica ni radiológica de fracaso en la fusión ósea $(p=1)$. Esto resulta llamativo si se considera que el grupo con FCP presentaba mayor prevalencia de antecedentes que podrían dar lugar a una mala consolidación del callo óseo como tabaquismo (38,45\%), ingesta habitual de AINES $(61,54 \%)$ y osteoporosis $(7,69 \%)$, y a su vez fue el grupo que presentó mayor número de artrodesis instrumentadas lumbares superiores a los dos niveles (53,85\%).

\section{CONCLUSIÓN}

Nuestra experiencia temprana con el uso de FCP enriqueciendo al injerto óseo en artrodesis instrumentadas lumbares posterolaterales no mostraría un claro beneficio en el uso de los mismos teniendo en cuenta que, si bien en el grupo de pacientes en los que fueron aplicados no hubo incidencia de pseudoartrosis, fue el grupo que registró mayor número de complicaciones postoperatorias y menor porcentaje de fusiones radiológicamente sólidas en comparación con la muestra de pacientes en la que los FCP no se utilizaron.

A pesar de que el número total de pacientes en el presente estudio no permite la obtención de resultados estadísticamente significativos, el presente puede ser el inicio de un estudio mayor, quizás debiendo aplicar a un análisis multicéntrico que permita una muestra significativa. crecimiento. Rev Asoc Argent Ortop Traumatol. 2007;72(4):373-81.

6. Lind M. Growth factor stimulation of bone healing. Effects on osteoblasts, osteomies, and implants fixation. Acta Orthop Scand Suppl. 1998;283:2-37.

7. Lowery GL, Kulkarni S, Pennisi AE. Use of autologous growth factors in lumbar spinal fusion. Bone. 1999:25(Suppl 2):47S-50S

8. Bose B, Balzarini MA. Bone graft gel: autologous growth factors used with autograft bone for lumbar spine fusions. Adv Ther. 2002;19(4):170-5 